\title{
Characteristic Analysis of Spreading Waveform of System Dynamics Based on MySQL Database
}

\author{
Kaihua $\mathrm{Xu}^{1}$, Yali $\mathrm{Xu}{ }^{2}$ \\ ${ }^{1}$ Police Training Management Department, Jiangxi People's Police College, Nanchang, 330103, \\ China \\ ${ }^{2}$ School of Physical Education, Jiangxi University of Traditional Chinese Medicine, Nanchang, \\ 330004, China \\ kaihua_xu@yeah.net
}

Keywords: System dynamics, Group emotion, Apriori algorithm, Association data, MySQL database.

\begin{abstract}
In order to study internal drive force, negative group emotion growth and diffusion of politics officers, this paper designs the emotional propagation analysis model. The model uses the frequent sets characteristic of Apriori algorithm database to connect the emotional characteristics data, and gets the confidence interval of the data association. This paper uses MySQL to establish the emotional characteristics database of law enforcement and police officers, and extracts the emotional data feature. Through calculating, this paper gets the emotional propagation characteristic distribution curve, and gets the emotional propagation validity curve under different number of person, which verified the effectiveness and reliability of dynamics system in the study of emotional propagation for law enforcement officers, which provides a new computer method for the study on group emotion.
\end{abstract}

\section{Introduction}

With the acceleration of economic development and social transformation, social contradictions become increasingly prominent, and the group incidents of social concern has caused a large amount of related research. People mainly focus on dealing with group events and post intervention $[1,2]$. Group event is the behavior characterization of group emotional, the latter is the internal driving force of the former, and the former is the power source of the negative group emotion growth and diffusion. The so-called group emotion refers to when people belong to a group and produce the recognition on this group, and they will make emotional reaction to the events affecting the group. This paper establishes a system dynamics database model of group emotional propagation, and uses the relevance algorithm to extract feature data. The general framework is as follows:

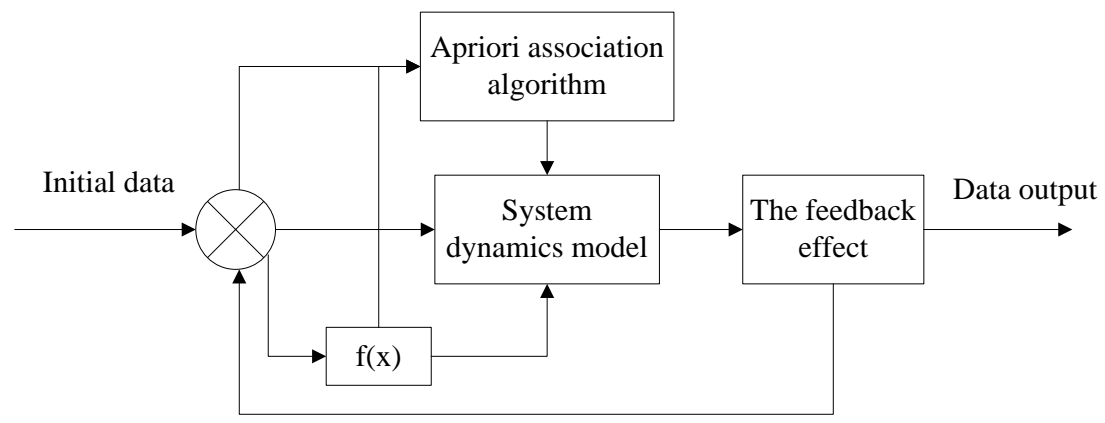

Fig.1: The system dynamics model of group emotion characteristics propagation

Figure 1 shows the system dynamics model of group emotion characteristics propagation [3]. The design framework is mainly based on the Apriori association algorithm to extract emotional drive data and feedback adjustment according to the data features, finally gets the psychological characteristics MySQL database. 


\section{Algorithm Design of Group Emotional Propagation Model}

The group emotional propagation model generally includes a variety of emotional characteristics data, each emotion feature data contains a variety of information, so it is necessary to establish a feature database to extract emotional propagation model [4-6]. First it defines emotional data information, as shown in Figure 2.

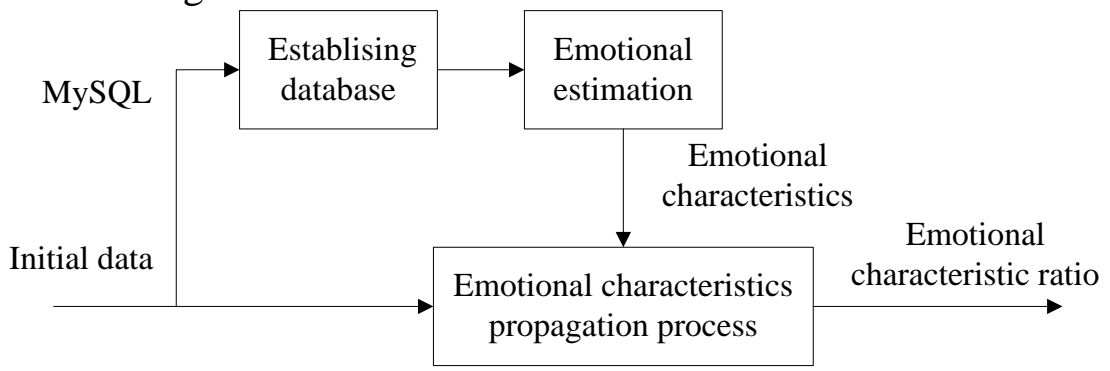

Fig.2: Group emotion data extraction

As shown in Figure 2, suppose that a packet has $i$ kind of emotional feature information, and then the character information can be defined as $C_{j}(j=1,2, \cdots, i)$. The emotional characteristics of data, $m^{*} n$ data package $C_{B}(j)$ is a $j$ dimensional vector, and its expression is as shown in formula (1).

$$
C_{B}(j)=\frac{p C_{j}}{m \times n} .
$$

$p C_{j}$ is the emotional integer in emotion characteristics. In order to establish feature relevance of emotional data, this paper establishes a emotional events, such as negative emotion work event $S$, association rules $E \Rightarrow F$ refers to the percentage of $E$ and $F$ in the total number of event, as shown in formula (2).

$$
\sup p(E \Rightarrow F)=\frac{|\{S: E \cup F \subseteq S, S \in D\}|}{|D|} \text {. }
$$

And the confidence can use formula (3) to expresses; it refers to the percentage of $E$ and $F$ in the only supporting $E$ event.

$$
\operatorname{conf}(E \Rightarrow F)=\frac{|\{S: E \cup F \subseteq S, S \in D\}|}{|S: E \subseteq S, S \in D|} .
$$

Priori algorithm is mainly to use the frequent sets characteristic in application database, and it uses $k$ item sets to find the $k+1$ set, and uses $L_{i-1} \times L_{i-1}$ to connect candidate sets $A_{i}$. If $l_{k}(j)$ is an item set of $L_{i-1}$, so:

$$
l_{1}[1]=l_{2}[1] \wedge l_{1}[2]=l_{2}[2] \cdots\left(l_{1}[k-1]=l_{2}[k-1]\right) .
$$

So connecting $l_{1}, l_{2}$, it can get $l_{1}[1], l_{2}[1] \cdots\left(l_{1}[k-1], l_{2}[k-1]\right)$.

$$
l_{1}[k-1]=l_{2}[k-1] \text {. }
$$

To get $l_{1}, l_{2}$, the formula (5) can be repeated.. This paper uses the MySQL database to establish emotional characteristic database of politics and law police officers. And the main MySQL program is as follows:

drop database if exists school;

create database school;

use school;

create table teacher

(id int(3) auto_increment not null primary key,

name char(10) not null,

address varchar(50) default 'emotion',

year date 
);

insert into teacher values(',' Individual emotional characteristics ', 'Law enforcement officers, insert into teacher values(',' Group emotional characteristics ', ' Law enforcement officers,

\section{Simulation Analysis of Group Emotional Propagation Model}

In order to effectively verify the effectiveness and reliability of system dynamics model of politics and law police officers group emotion designed in second section, this paper uses the MySQL database to establish the characteristic database of group emotion, and extracts the data feature, finally gets the general rule of group emotional propagation.

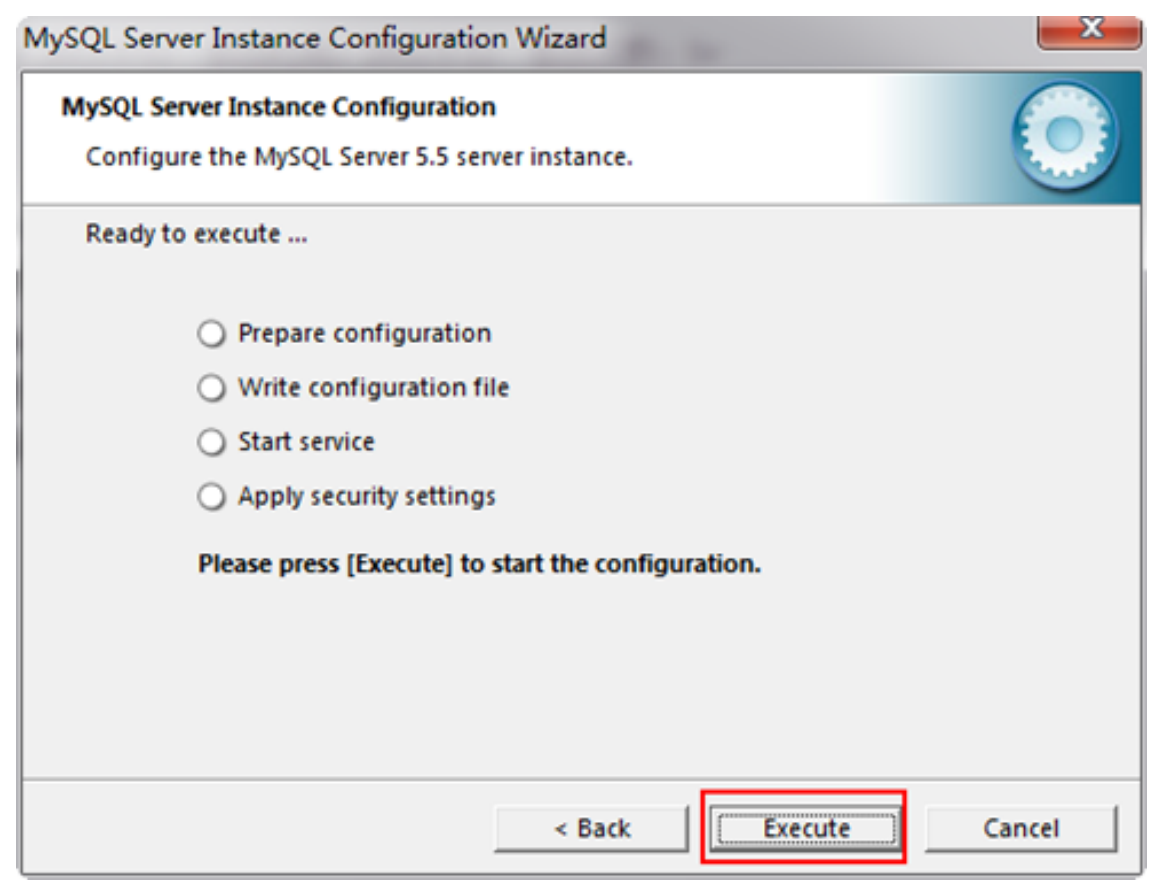

Fig.3: MySQL database data export

Figure 3 shows the schematic diagram of MySQL database data export [7]. MySQL is a relational database management system with open source code, and the original developer is Swedish MySQL AB Company, which is acquainted by Sun Microsystems in 2008.

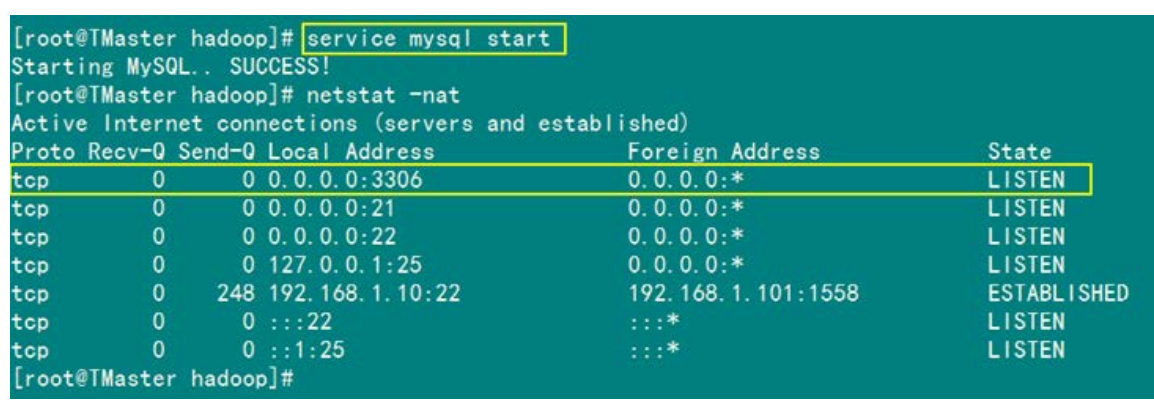

Fig.4: Preparation of politics and law police officers group emotional MySQL database

Figure 4 shows the preparation of politics and law police officers group emotional MySQL database. Under the group working state of politics and law police officers, group psychology shows certain correlation characteristics [8]. According to different IP psychology access methods, it encodes the different and similar psychological features data, and obtains the group psychological feature model as shown in Figure 5. 


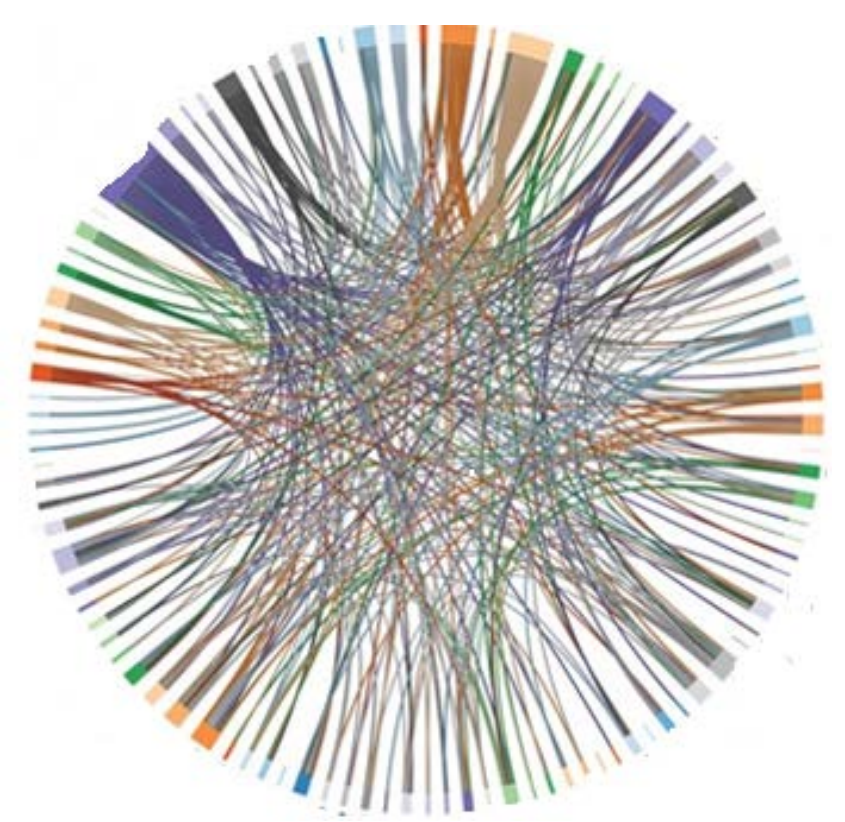

Fig.5: Group emotional propagation simulation model

Figure 5 shows the simulation model of the politics and law police officers emotional propagation. From the chart it can be seen, the emotional propagation model divided by group emotional database has a strong correlation, and the correlation strength is different [9]. From green to orange, it represents from strong to weak of group emotions.

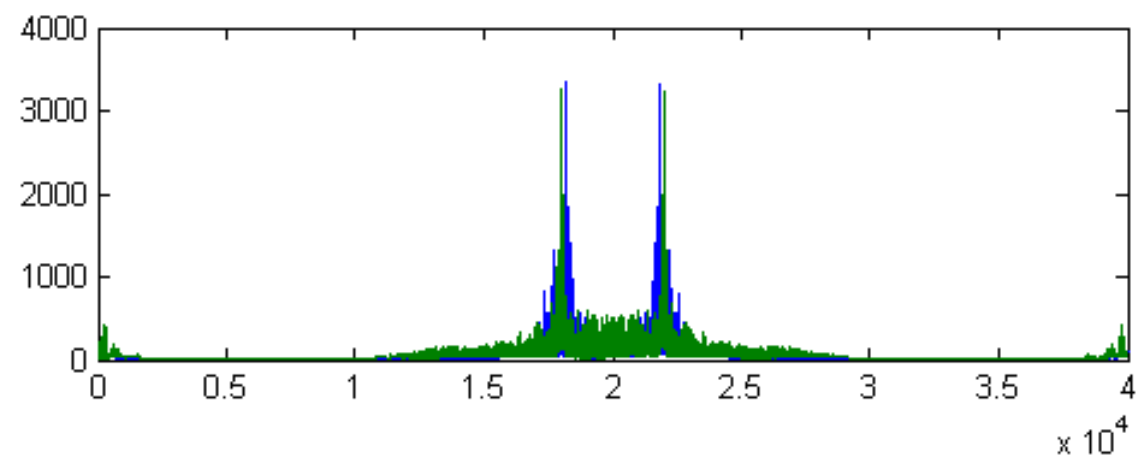

Fig.6: Analysis of emotional feature data

Figure 6 shows the group emotional propagation extraction results for politics and law police officers. From the chart it can be seen, in the 10000 data samples, emotional characteristics are mainly embodied in the job performance evaluation and negative work, that means that the two are fastest spreading group emotional characteristics data.

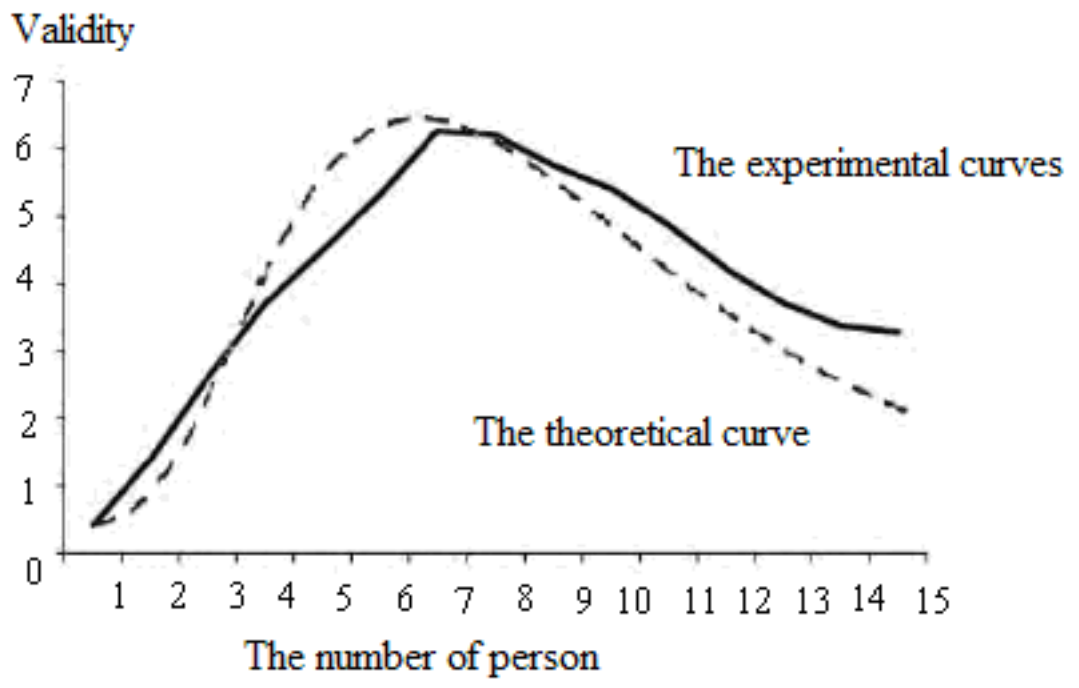

Fig.7: Propagation validity curves for different number of person 
Figure 7 shows the propagation validity curves for different number of person. From the chart it can be seen, the number of person has bigger influence on emotional communication, and the maximum propagation validity is concentration on 6-8 [10]. The simulation result is close to theoretical result, which shows that the simulation is reliable.

\section{Summary}

Based on the internal driving system dynamics theory of group emotion, combined with the emotional data characteristics, this paper designs MySQL database model of emotional feature analysis, and does correlation operation on the data, finally gets the negative emotion propagation data curve under the influence of emotion characteristics. This paper uses the Apriori algorithm to design the frequent set feature database, and does relevance feature extraction on the emotion. The relevance confidence interval is obtained, and it is applied in the emotional propagation evaluation of law enforcement and police officers. By calculating this paper gets the distribution characteristic curve of emotional propagation and emotional propagation validity curves for different persons, which is a new group emotional propagation analysis method. But this approach only uses the system dynamics method from the emotional propagation internal driving force, and it can study more system dynamics theory from the emotion in the future.

\section{Acknowledgments}

The work was supported by the Twelfth-five Plan Project of Education Science in Jiangxi Province with the project number 11YB425 and the project name Empirical Research on How the Heart Behavior Training Can Improve the Mental Health Level of the Policemen of Politics and Law.

\section{References}

[1] S.W. Zhang, E.P. Wang. The mobilization and organization mechanism of cluster behavior in the group event. Advances in psychological science, 2012, 4(12): 10-13.

[2] X. Wang, W.Z. Li, J.G. Du. A review of emotional contagion theoretical. The progress in psychological science, 2013, 2(8): 112-115.

[3] F. Liu, B. Zuo. Intergroup emotions theory and research. Advances in psychological science, 2012, 4(6): 61-64.

[4] Q. Zhan, J.P. Feng, E.P. Wang. Intergroup threat classification and its effect on group prejudice. Advances in psychological science, 2013, 4(2): 42-45.

[5] G. Li, P. Fang Y. Jiang. Current status and research on emotional reaction time power. Advances in psychological science, 2012, 6(1): 56-59.

[6] M.N. Wan, L. Liu, J. Qiu, X.L. Yang. The cluster behavior definition, psychological mechanism and behavior measuring. Advances in psychological science, 20125, 3(5): 32-35.

[7] C.F. Wang. Rumor spreading model and digestion for public emergency. Modern communication (Journal of Communication University of China), 2012, 4(6): 62-645.

[8] Y. Bai, J.L. Liu. Study on the dynamic effect of word and mouth communication: a theoretical model. Statistics and decision, 2013, 5(16): 62-68.

[9] S.Q. Ma, C. Jiao can, M.Q. Zhang. Application of social network analysis in the psychological study. Advances in psychological science, 2013, 5(5): 21-24.

[10] Z.R. Yu. The main type and its basic characteristics of the current group incidents in China. Journal of China University of Political Science and Law, 2013, 4(6): 78-81. 